\title{
Secure Communications via Cooperative Jamming in Two-hop Relay Systems
}

\author{
Jing Huang and A. Lee Swindlehurst \\ Electrical Engineering and Computer Science \\ University of California, Irvine, CA 92697 \\ Email: \{jing.huang; swindle\}@uci.edu
}

\begin{abstract}
This paper proposes a cooperative jamming strategy for two-hop relay networks where the eavesdropper can wiretap the relay channels in both hops. The problems of jamming beamformer design and power allocation are investigated jointly for two scenarios where the eavesdropper has either a single or multiple antennas, with the assumption that the global channel state information (CSI) is available. Under a constraint that the jamming signal lies in a subspaces orthogonal to the channels to legitimate nodes, we derive closed-form solutions for the jamming beamformers. Based on these results, we find the optimal solution for power allocation via geometric programming.
\end{abstract}

\section{INTRODUCTION}

Wireless networks are inherently vulnerable to eavesdropping. Formal study of this problem, referred to as the "wiretap" channel, was first introduced by Wyner in [1] and the secrecy capacity was defined as the maximal achievable rate that guaranteed secrecy about the information transmitted from the source to its intended receiver. Recently, there has been considerable interest in examining secrecy in multiple-input multiple-output (MIMO) systems [2], [3]. With the additional degrees of freedom provided by the extra antennas, transmitters can degrade the channel condition of the eavesdroppers while maintaining good channel quality to legitimate users by proper beamformer design or through the use of jamming [4][6].

As a natural extension, wiretap channels have also been investigated in cooperative relaying networks [4], [7], [8]. In these cases, relays or even the signal destinations themselves can be used as helpers to provide additional jamming to confuse the eavesdropper. This approach can be thought of as cooperative jamming. In [9], a four-terminal relayeavesdropper channel is introduced and a noise-forwarding strategy is devised to degrade the eavesdropper's signal. A two-stage cooperative jamming protocol is investigated in [4], although the "relays" only play the role of helpers and do not actually relay the information signals. In [10], a system design is proposed for a decode-and-forward relay network. However, this work only considers the scenario where the eavesdropper cannot wiretap the first-hop channel between the source and relay.

This paper proposes a cooperative jamming strategy for a two-hop wireless relay system in which the eavesdropper can wiretap the relay channels for both hops. The overall performance of the system over both hops is considered rather than one hop at a time, since the latter case is similar to the conventional MIMO wiretap channel. We focus on the scenario where a direct link cannot be established between the source and destination node due to the power constraint. Both the source and destination nodes are assumed to have multiple antennas, and both provide jamming signals in order to guarantee that the eavesdropper is degraded whether or not it is close to the source or the destination. We investigate the joint design of the jamming beamformers and the power allocation for the following two cases: (1) maximizing the secrecy capacity with certain power constraints, and (2) minimizing the transmit power with a fixed target secrecy capacity. Since joint optimization of the beamformers and power allocation is in general intractable, even if global channel state information (CSI) is available, we use a suboptimal constraint that forces the jamming signals to lie in a subspace orthogonal to the channels to legitimate nodes, which allows us to derive closedform solutions for the jamming beamformers. Based on these results, we find the optimal power allocation using the method of geometric programming. We assume that global CSI is available to all nodes in the system, and include performance comparisons for the case where eavesdropper's CSI is unknown.

\section{SySTEM MODEL}

We consider a two-hop four-terminal relay system composed of a source (Alice), a destination (Bob), a relay node and an eavesdropper (Eve), as shown in Fig. 1. All nodes are assumed to be half-duplex. Alice and Bob possess multiple antennas, the number of which we denote by $M$ and $N$, respectively. Eve may either have one antenna $\left(N_{e}=1\right)$ or multiple antennas $\left(N_{e}>1\right)$; both scenarios are considered separately in the paper. The relay is assumed to have a single antenna and Eve is assumed to be able to wiretap both hops. The key idea is that Alice and Bob can help form a cooperative jamming system without the addition of extra helpers outside the two-hop relay system. All channels are assumed to be flat fading, and a decode-and-forward (DF) relay is used. In the first hop, Alice transmits the information signal to the Relay, and Bob transmits a jamming signal at the same time. The signals received by the Relay and Eve during the first hop can be expressed as

$$
\begin{gathered}
y_{r}=\mathbf{h}_{a r} \mathbf{t}_{a} z_{a}+\mathbf{h}_{b r} \mathbf{w}_{b} z_{b}^{\prime}+n_{r} \\
\mathbf{y}_{e 1}=\mathbf{H}_{a e} \mathbf{t}_{a} z_{a}+\mathbf{H}_{b e} \mathbf{w}_{b} z_{b}^{\prime}+\mathbf{n}_{e 1},
\end{gathered}
$$




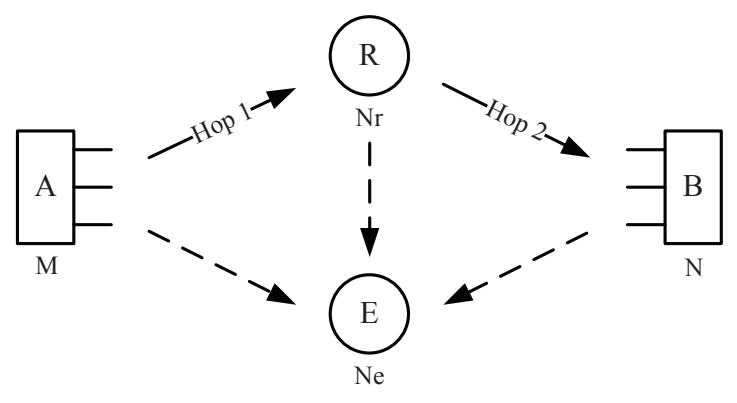

Fig. 1. System model.

where $z_{a}$ is the information signal transmitted by Alice, $z_{b}^{\prime}$ is the jamming signal transmitted by Bob, $\mathbf{t}_{a}$ is the $M \times 1$ transmit beamformer used by Alice, $\mathbf{w}_{b}$ is the $N \times 1$ jamming beamformer employed by Bob, $n_{r}$ and $\mathbf{n}_{e 1}$ represent naturally occuring noise and interference at the Relay and Eve, respectively, $\mathbf{H}_{i j}\left(\mathbf{h}_{i j}\right)$ is the channel matrix from $i$ to $j$, with $i, j \in\{a, b, e, r\}$ denoting which of the four terminals is involved. The power allocation is denoted as $\mathbb{E}\left[\left|z_{a}\right|^{2}\right]=p_{s}$ and $\mathbb{E}\left[\left|z_{b}^{\prime}\right|^{2}\right]=p_{b}$. For simplicity, we assume that the noise at all nodes is spatially white Gaussian noise with the same variance $\sigma^{2}$.

In the second hop, the Relay forwards the information signal to Bob while Alice jams, and thus the signals received by Bob and Eve are

$$
\begin{gathered}
y_{b}=\mathbf{t}_{b}^{H} \mathbf{h}_{r b} z_{r}+n_{b} \\
\mathbf{y}_{e 2}=\mathbf{h}_{r e} z_{r}+\mathbf{H}_{a e} \mathbf{w}_{a} z_{a}^{\prime}+\mathbf{n}_{e 2},
\end{gathered}
$$

where $z_{r}$ is the information signal transmitted by the Relay, $z_{a}^{\prime}$ is the jamming signal transmitted by Alice, $\mathbf{t}_{b}$ is the $N \times 1$ transmit beamformer used by Bob, $\mathbf{w}_{a}$ is the $M \times 1$ jamming beamformer employed by Alice, $n_{b}, \mathbf{n}_{e 2}$ represent the noise at Bob and Eve, and $(\cdot)^{H}$ represents the Hermitian transpose. The power allocation for this hop is $\mathbb{E}\left[\left|z_{r}\right|^{2}\right]=p_{r}, \mathbb{E}\left[\left|z_{a}^{\prime}\right|^{2}\right]=p_{a}$.

For the transmit beamformer $\mathbf{t}_{a}$ in the first hop, we choose the generalized eigenvector of the pencil $\left(\mathbf{h}_{a r}^{H} \mathbf{h}_{a r}, \mathbf{H}_{a e}^{H} \mathbf{H}_{a e}\right)$ with the largest generalized eigenvalue, which achieves the secrecy capacity for the single-hop wiretap channel in the high SNR regime [6]. In the second hop, since Bob will not see interference from Alice's jamming signal due to the assumption that Alice has no direct link to Bob, the best receive beamformer is the maximal ratio beamformer $\mathbf{t}_{b}=\frac{\mathbf{h}_{a r}^{H}}{\left\|\mathbf{h}_{a r}^{H}\right\|}$.

\section{Cooperative JAMming FOR Relaying Channels}

In this section, we will discuss the design of the jamming beamformers and power allocation for cases where $N_{e}=1$ and $N_{e}>1$. Both global power constraints $\left(p_{s}+p_{b} \leq P\right.$ in the first hop and $p_{r}+p_{a} \leq P$ in the second hop) and individual power constraints will be considered in these cases.

\section{A. Maximum Secrecy Capacity with Transmit Power Con- straint $\left(N_{e}=1\right)$}

For a two-hop DF-based relay channel, the capacity through the relay link can be written as [11]

$$
C_{r}=\frac{1}{2} \min \left\{\log _{2}\left(1+\gamma_{a r}\right), \log _{2}\left(1+\gamma_{r b}\right)\right\},
$$

where $\frac{1}{2}$ appears because the relay transmission is divided into two stages, and $\gamma_{i j}$ is the SINR for node $j$ and the signal from node $i$. Eve receives data during both hops, and the capacity of Eve can be expressed as

$$
C_{e}=\frac{1}{2} \log _{2}\left(1+\gamma_{a e}+\gamma_{r e}\right) .
$$

Therefore the secrecy capacity for a DF relaying wiretap channel can be written as

$$
C_{s}=\max \left\{0, C_{r}-C_{e}\right\} .
$$

Since the capacity of the relay link is limited by the SINR of the inferior hop, for a single data stream the transmit power for Alice and the Relay should be adjusted such that $\gamma_{a r}=\gamma_{r b}$. Since the Relay has only a single antenna, we can design $\mathbf{w}_{b}$ such that the jamming signals are completely nulled at the Relay, i.e., $\mathbf{h}_{b r} \mathbf{w}_{b}=0$. Therefore, the secrecy capacity can be written as

$$
\begin{aligned}
C_{s} & =\frac{1}{2} \log _{2} \frac{\left(1+\gamma_{a r}\right)}{\left(1+\gamma_{a e}+\gamma_{r e}\right)} \\
& =\frac{1}{2} \log _{2} \frac{\left(1+\frac{p_{s}\left|\mathbf{h}_{a r} \mathbf{t}_{a}\right|^{2}}{\sigma^{2}}\right)}{\left(1+\frac{p_{s}\left|\mathbf{h}_{a e} \mathbf{t}_{a}\right|^{2}}{p_{b}\left|\mathbf{h}_{b e} \mathbf{w}_{b}\right|^{2}+\sigma^{2}}+\frac{p_{r}\left|h_{r e}\right|^{2}}{p_{a}\left|\mathbf{h}_{a e} \mathbf{w}_{a}\right|^{2}+\sigma^{2}}\right)} .
\end{aligned}
$$

We aim to find the joint optimal solution for the jamming beamformers $\mathbf{w}_{a}, \mathbf{w}_{b}$, and the transmit power vector $\mathbf{p}(\mathbf{p}=$ $\left.\left\{p_{s}, p_{r}, p_{a}, p_{b}\right\}\right)$ in order to maximize the secrecy capacity $C_{s}$.

We first consider optimization of the jamming beamformers, which is straightforward. For $\mathbf{w}_{b}$, we want to maximize $\left|\mathbf{h}_{b e} \mathbf{w}_{b}\right|^{2}$, the contribution of the jamming signal at Eve, under the constraint that $\mathbf{h}_{b r} \mathbf{w}_{b}=0$. Defining $\mathbf{G}_{b}^{\perp}=$ $\left[\mathbf{g}_{b 1}, \mathbf{g}_{b 2}, \ldots, \mathbf{g}_{b(m-1)}\right]$ as the orthonormal basis of the space orthogonal to $\operatorname{span}\left\{\mathbf{h}_{b r}^{H}\right\}$, the jamming beamformer from Bob can be written as $\mathbf{w}_{b}=\mathbf{G}_{b}^{\perp} \mathbf{c}_{b}$, for some unit-length vector $\mathbf{c}_{b}$. The optimization problem is then formulated as

$$
\begin{array}{ll}
\max _{\mathbf{c}_{b}} & \mathbf{c}_{b}^{H} \mathbf{G}_{b}^{\perp{ }^{H}} \mathbf{h}_{b e}^{H} \mathbf{h}_{b e} \mathbf{G}_{b}^{\perp} \mathbf{c}_{b} \\
\text { s.t. } & \mathbf{c}_{b}^{H} \mathbf{c}_{b}=1 .
\end{array}
$$

and the solution is simply $\mathbf{c}_{b}=\frac{\mathbf{G}_{b}^{\perp H} \mathbf{h}_{b e}^{H}}{\left\|\mathbf{G}_{b}^{\perp H} \mathbf{h}_{b e}^{H}\right\|}$. For $\mathbf{w}_{a}$, since the Relay is half-duplex, we maximize $\left|\mathbf{h}_{a e} \mathbf{w}_{a}\right|^{2}$ such that $\mathbf{w}_{a}^{H} \mathbf{w}_{a}=1$. The solution is thus simply $\mathbf{w}_{a}=\frac{\mathbf{h}_{a e}^{H}}{\left\|\mathbf{h}_{a e}^{H}\right\|}$.

Next we find the power allocation that maximizes the secrecy capacity. It is difficult to test the Hessian matrix in (8) for convexity, so we seek to solve the problem by maximizing the lower bound of $C_{s}$. Utilizing the beamformers derived 
above and according to (8), the secrecy capacity can be lower bounded as

$$
\begin{aligned}
C_{s}(\mathbf{p}) & \geq \frac{1}{2} \log _{2} \frac{\gamma_{a r}}{\left(1+\gamma_{a e}+\gamma_{r e}\right)} \\
& =\frac{1}{2} \log _{2} \frac{\frac{p_{s}\left|\mathbf{h}_{a r} \mathbf{t}_{a}\right|^{2}}{\sigma^{2}}}{\left(1+\frac{p_{s}\left|\mathbf{h}_{a e} \mathbf{t}_{a}\right|^{2}}{\tilde{p_{b}}}+\frac{p_{r}\left|h_{r e}\right|^{2}}{\tilde{p_{a}}}\right)} \\
& =\frac{1}{2} \log _{2} \frac{\left|\mathbf{h}_{a r} \mathbf{t}_{a}\right|^{2} / \sigma^{2}}{f(\mathbf{p})},
\end{aligned}
$$

where $\tilde{p_{b}}=p_{b}\left|\mathbf{h}_{b e} \mathbf{w}_{b}\right|^{2}+\sigma^{2}, \tilde{p_{a}}=p_{a}\left|\mathbf{h}_{a e} \mathbf{w}_{a}\right|^{2}+\sigma^{2}$. Thus, maximization of the secrecy capacity is equivalent to minimizing $f(\mathbf{p})$ :

$$
\begin{aligned}
\min _{\mathbf{p}} & c_{1} p_{s}^{-1}+c_{2} \tilde{p}_{b}^{-1}+c_{3} p_{s}^{-1} p_{r} \tilde{p}_{a}^{-1} \\
\text { s.t. } & p_{s}+\left|\mathbf{h}_{b e} \mathbf{w}_{b}\right|^{-2} \tilde{p_{b}} \leq P+\left|\mathbf{h}_{b e} \mathbf{w}_{b}\right|^{-2} \sigma^{2} \\
& p_{r}+\left|\mathbf{h}_{a e} \mathbf{w}_{a}\right|^{-2} \tilde{p_{a}} \leq P+\left|\mathbf{h}_{a e} \mathbf{w}_{a}\right|^{-2} \sigma^{2}
\end{aligned}
$$

where (12) and (13) are the global transmit power constraints derived from $p_{s}+p_{b} \leq P$ and $p_{r}+p_{a} \leq P$, and $\mathbf{c}=$ $\left\{1,\left|\mathbf{h}_{a e} \mathbf{t}_{a}\right|^{2},\left|h_{r e}\right|^{2}\right\}$.

The optimization problem stated above is in the standard form for Geometric Programming (GP) problems, and (11)(13) are posynomials of GP. GP is a class of non-linear optimization problems that can be readily turned into convex optimization problems, and hence a global optimum can be computed efficiently [12]. For individual transmit power constraints, we can similarly form the optimization problem as

$$
\begin{array}{ll}
\min _{\mathbf{p}} & c_{1} p_{s}^{-1}+c_{2}{\tilde{p_{b}}}^{-1}+c_{3} p_{s}^{-1} p_{r}{\tilde{p_{a}}}^{-1} \\
\text { s.t. } & p_{s} \leq P_{1}, p_{r} \leq P_{2} \\
& \tilde{p_{a}} \leq P_{3}\left|\mathbf{h}_{b e} \mathbf{w}_{b}\right|^{2}+\sigma^{2}, \tilde{p_{b}} \leq P_{4}\left|\mathbf{h}_{a e} \mathbf{w}_{a}\right|^{2}+\sigma^{2}
\end{array}
$$

where $P_{i}, i=1, \cdots, 4$ are the individual power constraints for each node, and (14) is derived from $p_{b} \leq P_{3}$ and $p_{a} \leq P_{4}$.

\section{B. Minimum Transmit Power with Fixed Secrecy Capacity $\left(N_{e}=1\right)$}

For the minimum jamming power problem, we still choose jamming beamformers that lie in the subspace orthogonal to the intended channels. Thus for the first hop, as before we will have $\mathbf{w}_{b}=\rho_{b} \mathbf{G}_{\mathbf{b}}^{\perp} \mathbf{e}_{b}$, where $\mathbf{e}_{b}$ is the coefficient vector, and $\rho_{b}$ is a scalar that maintains the unit norm of $\mathbf{w}_{b}$. We aim to minimize the norm of $\mathbf{e}_{b}$ under a fixed target secrecy capacity $C_{0}$. According to (10), the problem can be formulated as

$$
\begin{array}{ll}
\min _{\mathbf{e}_{b}} & \mathbf{e}_{b}^{H} \mathbf{e}_{b} \\
\text { s.t. } & \mathbf{e}_{b}^{H} \mathbf{G}_{b}^{\perp^{H}} \mathbf{h}_{b e}^{H} \mathbf{h}_{b e} \mathbf{G}_{b}^{\perp} \mathbf{e}_{b} \geq k\left(C_{0}, \mathbf{w}_{a}\right),
\end{array}
$$

where $k\left(C_{0}, \mathbf{w}_{a}\right)$ is a function of $C_{0}$ and $\mathbf{w}_{a}$ independent of $\mathbf{w}_{b}$. The solution is again seen to be the eigenvector of $\mathbf{G}_{b}^{\perp H} \mathbf{h}_{b e}^{H} \mathbf{h}_{b e} \mathbf{G}_{b}^{\perp}$ corresponding to the largest eigenvalue. Since it is a rank one Hermitian matrix, the result can be explicitly presented as $\mathbf{w}_{b}=\mathbf{G}_{b}^{\perp} \frac{\mathbf{G}_{b}^{\perp H} \mathbf{h}_{b e}^{H}}{\left\|\mathbf{G}_{b}^{\perp H} \mathbf{h}_{b e}^{H}\right\|}$, which is the same result as in the capacity maximization problem. Similarly for the second hop, we have $\mathbf{w}_{a}=\frac{\mathbf{h}_{a e}^{H}}{\left\|\mathbf{h}_{a e}^{H}\right\|}$. Considering the transmit power of all the nodes, we can now formulate the optimization problem under the global transmit power constraint as

$$
\begin{array}{ll}
\min _{\mathbf{p}} & \max \left(p_{s}+\tilde{p_{b}}, p_{r}+\tilde{p_{a}}\right) \\
\text { s.t. } & f(\mathbf{p}) \leq \frac{\left|\mathbf{h}_{a r} \mathbf{t}_{a}\right|^{2}}{2^{2 C_{0}} \sigma^{2}}
\end{array}
$$

where $f(\mathbf{p})$ is given in (10). The above problem is a generalized GP where (15) and (16) are the generalized posynomials. If individual power constraints are used, (15) should be rewritten as $\min _{\mathbf{p}} \max \left(p_{s}, \tilde{p_{b}}, p_{r}, \tilde{p_{a}}\right)$ instead.

\section{Maximum Secrecy Capacity with transmit power constraint $\left(N_{e}>1\right)$}

In this section, we consider the scenario where Eve is equipped with multiple antennas. In this case, Eve would use beamformers $\mathbf{w}_{e 1}$ and $\mathbf{w}_{e 2}$ to receive signals from Alice and the Relay. Thus we have

$$
\mathbf{w}_{e 1}^{H} \mathbf{y}_{e 1}=\mathbf{w}_{e 1}^{H}\left(\mathbf{H}_{a e} \mathbf{t}_{a} z_{a}+\mathbf{H}_{b e} \mathbf{w}_{b} z_{b}^{\prime}+\mathbf{n}_{e 1}\right) .
$$

Assuming Eve can compute the beamformer which yields the best SINR,

$$
\mathbf{w}_{e 1}=\left(p_{b} \mathbf{H}_{b e} \mathbf{w}_{b} \mathbf{w}_{b}^{H} \mathbf{H}_{b e}^{H}+\sigma^{2} \mathbf{I}\right)^{-1} \mathbf{H}_{a e} \mathbf{t}_{a},
$$

and the resulting SINR for Eve during the first hop is

$$
\begin{aligned}
& \gamma_{a e}=p_{s} \mathbf{t}_{a}^{H} \mathbf{H}_{a e}^{H}\left(p_{b} \mathbf{H}_{b e} \mathbf{w}_{b} \mathbf{w}_{b}^{H} \mathbf{H}_{b e}^{H}+\sigma^{2} \mathbf{I}\right)^{-1} \mathbf{H}_{a e} \mathbf{t}_{a} \\
& =p_{s}\left(\mathbf{t}_{a}^{H} \mathbf{H}_{a e}^{H} \mathbf{H}_{a e} \mathbf{t}_{a}-\frac{\mathbf{t}_{a}^{H} \mathbf{H}_{a e}^{H} \mathbf{H}_{b e} \mathbf{w}_{b} \mathbf{w}_{b}^{H} \mathbf{H}_{b e}^{H} \mathbf{H}_{a e} \mathbf{t}_{a}}{\mathbf{w}_{b}^{H}\left(\frac{\sigma^{2}}{p_{b}} \mathbf{I}+\mathbf{H}_{b e}^{H} \mathbf{H}_{b e}\right) \mathbf{w}_{b}}\right),
\end{aligned}
$$

where (19) holds due to $\left(\mathbf{I}+\mathbf{A} \mathbf{A}^{H}\right)^{-1}=\mathbf{I}-\mathbf{A}(\mathbf{I}+$ $\left.\mathbf{A}^{H} \mathbf{A}\right)^{-1} \mathbf{A}^{H}$. As before, to maintain jamming with zero interference at the relay, Bob's jamming beamformer will be given by $\mathbf{w}_{b}=\mathbf{G}_{b}^{\perp} \mathbf{c}_{b}$ with $\left\|\mathbf{c}_{b}\right\|=1$, and the optimization issue becomes

$$
\begin{aligned}
\max _{\mathbf{c}_{b}} & \frac{\mathbf{c}_{b}^{H} \mathbf{a}_{b} \mathbf{a}_{b}^{H} \mathbf{c}_{b}}{\mathbf{c}_{b}^{H}\left(\frac{\sigma^{2}}{p_{b}} \mathbf{I}+\mathbf{B}_{b}^{H} \mathbf{B}_{b}\right) \mathbf{c}_{b}} \\
\text { s.t. } & \mathbf{c}_{b}^{H} \mathbf{c}_{b}=1,
\end{aligned}
$$

where $\mathbf{a}_{b}=\mathbf{G}_{b}^{\perp H} \mathbf{H}_{b e}^{H} \mathbf{H}_{a e} \mathbf{t}_{a}$ and $\mathbf{B}_{b}=\mathbf{H}_{b e} \mathbf{G}_{b}^{\perp}$. The maximum value of the Rayleigh quotient in (20) is the largest generalized eigenvalue of the matrix pencil $\left(\mathbf{a}_{b} \mathbf{a}_{b}^{H}, \frac{\sigma^{2}}{p_{b}} \mathbf{I}+\mathbf{B}_{b}^{H} \mathbf{B}_{b}\right)$, and the vector that achieves it is the corresponding generalized eigenvector. Since $\mathbf{a}_{b} \mathbf{a}_{b}^{H}$ is rank one, the solution can be written as

$$
\mathbf{w}_{b}=\mathbf{G}_{b}^{\perp} \frac{\left(\frac{\sigma^{2}}{p_{b}} \mathbf{I}+\mathbf{B}_{b}^{H} \mathbf{B}_{b}\right)^{-1} \mathbf{a}_{b}}{\left\|\left(\frac{\sigma^{2}}{p_{b}} \mathbf{I}+\mathbf{B}_{b}^{H} \mathbf{B}_{b}\right)^{-1} \mathbf{a}_{b}\right\|},
$$

and $\gamma_{a e}$ becomes

$$
\gamma_{a e}=p_{s}\left(\mathbf{t}_{a}^{H} \mathbf{H}_{a e}^{H} \mathbf{H}_{a e} \mathbf{t}_{a}-\mathbf{a}_{b}^{H}\left(\frac{\sigma^{2}}{p_{b}} \mathbf{I}+\mathbf{B}_{b}^{H} \mathbf{B}_{b}\right)^{-1} \mathbf{a}_{b}\right) .
$$


Similarly for the second hop, we assume Eve employs the best beamformer,

$$
\mathbf{w}_{e 2}=\left(p_{a} \mathbf{H}_{a e} \mathbf{w}_{a} \mathbf{w}_{a}^{H} \mathbf{H}_{a e}^{H}+\sigma^{2} \mathbf{I}\right)^{-1} \mathbf{h}_{r e},
$$

and the resulting SINR for Eve during the second hop becomes

$$
\begin{aligned}
\gamma_{r e} & =p_{r} \mathbf{h}_{r e}^{H}\left(p_{a} \mathbf{H}_{a e} \mathbf{w}_{a} \mathbf{w}_{a}^{H} \mathbf{H}_{a e}^{H}+\sigma^{2} \mathbf{I}\right)^{-1} \mathbf{h}_{r e} \\
& =p_{r}\left(\mathbf{h}_{r e}^{H} \mathbf{h}_{r e}-\frac{\mathbf{h}_{r e}^{H} \mathbf{H}_{a e} \mathbf{w}_{a} \mathbf{w}_{a}^{H} \mathbf{H}_{a e}^{H} \mathbf{h}_{r e}}{\mathbf{w}_{a}^{H}\left(\frac{\sigma^{2}}{p_{a}} \mathbf{I}+\mathbf{H}_{a e}^{H} \mathbf{H}_{a e}\right) \mathbf{w}_{a}}\right) .
\end{aligned}
$$

Using the same method as in (20), we have a similar result for the second hop:

$$
\mathbf{w}_{a}=\frac{\left(\frac{\sigma^{2}}{p_{a}}+\mathbf{H}_{a e}^{H} \mathbf{H}_{a e}\right)^{-1} \mathbf{H}_{a e}^{H} \mathbf{h}_{r e}}{\left\|\left(\frac{\sigma^{2}}{p_{a}} \mathbf{I}+\mathbf{H}_{a e}^{H} \mathbf{H}_{a e}\right)^{-1} \mathbf{H}_{a e}^{H} \mathbf{h}_{r e}\right\|},
$$

and $\gamma_{r e}$ becomes

$$
\gamma_{r e}=p_{r}\left(\mathbf{h}_{r e}^{H} \mathbf{h}_{r e}-\mathbf{h}_{r e}^{H} \mathbf{H}_{a e}\left(\frac{\sigma^{2}}{p_{a}} \mathbf{I}+\mathbf{H}_{a e}^{H} \mathbf{H}_{a e}\right)^{-1} \mathbf{H}_{a e}^{H} \mathbf{h}_{r e}\right) .
$$

Unlike the scenario with only one antenna at Eve, the jamming beamformers here are no longer independent of the jamming power, and thus we need to jointly optimize over both quantities.

Using the above analysis, the secrecy capacity can be lower bounded as

$$
\begin{aligned}
C_{s}(\mathbf{p}) & \geq \frac{1}{2} \log _{2} \frac{\gamma_{a r}}{\left(1+\gamma_{a e}+\gamma_{r e}\right)} \\
& =\frac{1}{2} \log _{2} \frac{\frac{p_{s}\left|\mathbf{h}_{a r} \mathbf{t}_{a}\right|^{2}}{\sigma^{2}}}{\left(1+\frac{p_{s}}{\tilde{p_{b}}}+\frac{p_{r}}{\tilde{p_{a}}}\right)} \\
& =\frac{1}{2} \log _{2} \frac{\left|\mathbf{h}_{a r} \mathbf{t}_{a}\right|^{2} / \sigma^{2}}{g(\mathbf{p})},
\end{aligned}
$$

where ${\tilde{p_{b}}}^{-1}=\mathbf{t}_{a}^{H} \mathbf{H}_{a e}^{H} \mathbf{H}_{a e} \mathbf{t}_{a}-\mathbf{a}_{b}^{H}\left(\frac{\sigma^{2}}{p_{b}} \mathbf{I}+\mathbf{B}_{b}^{H} \mathbf{B}_{b}\right)^{-1} \mathbf{a}_{b}$ and ${\tilde{p_{a}}}^{-1}=\mathbf{h}_{r e}^{H} \mathbf{h}_{r e}-\mathbf{h}_{r e}^{H} \mathbf{H}_{a e}\left(\frac{\sigma^{2}}{p_{a}} \mathbf{I}+\mathbf{H}_{a e}^{H} \mathbf{H}_{a e}\right)^{-1} \mathbf{H}_{a e}^{H} \mathbf{h}_{r e}$. In most cases of interest, $\tilde{p_{b}}$ and $\tilde{p_{a}}$ can be accurately approximated as linear functions of $p_{b}$ and $p_{a}$, which we denote by $\tilde{p_{b}}=$ $c_{1} p_{b}+c_{2}$ and $\tilde{p_{a}}=c_{3} p_{a}+c_{4}$. Using this approximation, the capacity maximization problem under a global power constraint $P$ becomes one of minimizing $g(\mathbf{p})$ in (25) as

$$
\begin{array}{cl}
\min _{\mathbf{p}} & p_{s}^{-1}+{\tilde{p_{b}}}^{-1}+p_{s}^{-1} p_{r}{\tilde{p_{a}}}^{-1} \\
\text { s.t. } & p_{s}+c_{1}^{-1} \tilde{p_{b}} \leq P+c_{1}^{-1} c_{2} \\
& p_{r}+c_{3}^{-1} \tilde{p_{a}} \leq P+c_{3}^{-1} c_{4}
\end{array}
$$

where the constraints are derived from $p_{s}+p_{b} \leq P$ and $p_{r}+$ $p_{a} \leq P$. This problem can also be efficiently solved with GP. If individual power constraints are employed, we have instead $p_{s} \leq P_{1}, p_{r} \leq P_{2}, c_{1}^{-1} \tilde{p_{b}} \leq P_{3}+c_{1}^{-1} c_{2}, c_{3}^{-1} \tilde{p_{a}} \leq P_{4}+c_{3}^{-1} c_{4}$.

\section{Minimum Transmit Power with Fixed Secrecy Capacity $\left(N_{e}>1\right)$}

Based on the discussion in Section III-B, it can be seen that minimizing transmit power is equivalent to the problem of maximizing the secrecy capacity under a certain power constraint. Therefore, we can still use the jamming beamformers

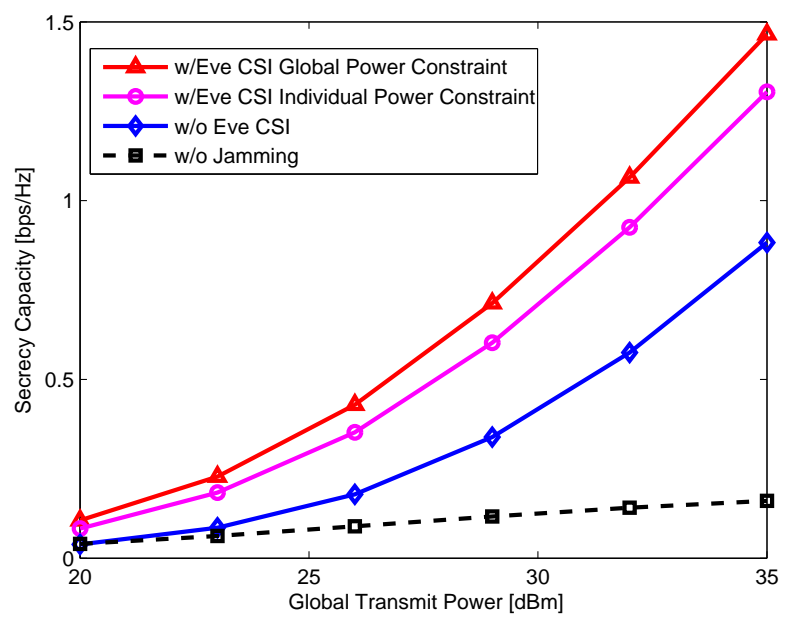

Fig. 2. Secrecy capacity vs. transmit power, $M=N=4, N_{e}=1, d_{a r}=$ $d_{r b}=500 \mathrm{~m}, d_{a e}=d_{b e}=400 \mathrm{~m}, d_{r e}=250 \mathrm{~m}$.

given in (21) and (24). Assuming $C_{0}$ is the target secrecy capacity to achieve, then according to (25) the problem of minimizing global transmit power is

$$
\begin{array}{ll}
\min _{\mathbf{p}} & \max \left(p_{s}+\tilde{p_{b}}, p_{r}+\tilde{p_{a}}\right) \\
\text { s.t. } & g(\mathbf{p}) \leq \frac{\left|\mathbf{h}_{a r} \mathbf{t}_{a}\right|^{2}}{2^{2 C_{0}} \sigma^{2}},
\end{array}
$$

where $g(\mathbf{p})$ is given in (25). To minimize individual transmit powers, (26) should be rewritten as $\min _{\mathbf{p}} \max \left(p_{s}, \tilde{p_{b}}, \tilde{p_{r}}, \tilde{p_{a}}\right)$ instead.

\section{NumericAl RESULts}

In the following simulations, we adopt a simple transmission model in which the channel gain is inversely proportional to the distance between two nodes. We assume a path-loss coefficient of 3.5, and the same background noise power $\sigma^{2}=-60 \mathrm{dBm}$ at all nodes. All results are calculated based on an average of 1000 independent trials.

In order to compare the performance of the different jamming schemes, we also investigate scenarios where Eve's CSI is unknown as well as conventional relay transmission schemes without jamming, besides the cooperative jamming discussed in previous sections. When Eve's CSI is unknown, we assume Alice and Bob uniformly spread the jamming power along all available spatial dimensions [5] and allocate half of the transmit power for signal and the other half for jamming. For the case of relaying without jamming, all the transmit power would be assigned to the signals of interest. For scenarios involving individual transmit power constraints, the total transmit power is assumed to be evenly distributed to all transmit nodes.

The secrecy capacity as a function of transmit power is shown in Fig. 2, where Alice and Bob both have four antennas and Eve has one. The distance between Alice and the Relay and between the Relay and Bob is taken to be $500 \mathrm{~m}$. Eve is assumed to be located $400 \mathrm{~m}$ from Alice and Bob, and $250 \mathrm{~m}$ 


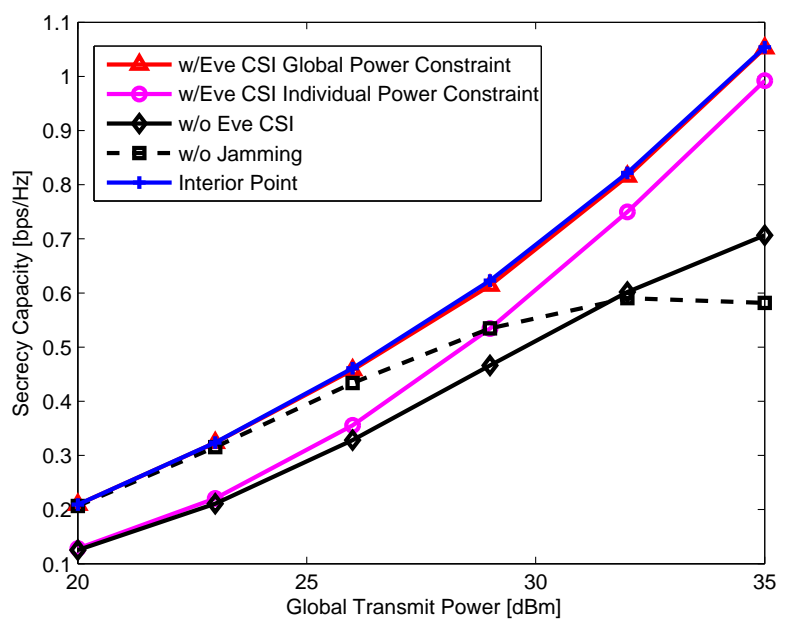

Fig. 3. Secrecy capacity vs. transmit power, $M=N=4, N_{e}=4, d_{a r}=$ $d_{r b}=500 \mathrm{~m}, d_{e}=1000 \mathrm{~m}$

from the Relay. In this case, schemes with cooperative jamming provide a significant improvement in secrecy capacity compared the traditional relaying scheme. The capacity of cooperative jamming with known CSI for the eavesdropper is higher than without CSI. Having a global power constraint yields slightly better performance than when individual power constraints are used due to the ability to efficiently allocate power for signal transmission and jamming.

Fig. 3 depicts the secrecy capacity when Eve has multiple antennas and is very far away from the relay link (including Alice, Bob and the Relay). In order to validate the performance of using GP to optimize the power allocation, we also have simulated the performance of the interior point method by carefully choosing reasonable initial points. It can be seen that our optimization results are very close to that of the interior point method. We can also see that when the achieved secrecy capacity is lower than $0.3 \mathrm{bps} / \mathrm{Hz}$, traditional relaying is almost as good as the cooperative jamming strategy. However, the capacity saturates at $0.6 \mathrm{bps} / \mathrm{Hz}$, while cooperative jamming can still achieve higher secrecy capacity as the transmit power is increased.

Fig. 4 presents the minimum transmit power as a function of Eve's position assuming a fixed target secrecy capacity of $0.5 \mathrm{bps} / \mathrm{Hz}$. In this case, Eve is equipped with multiple antennas and moves gradually away from the relay link and the power results are averaged in two hops. We see that when Eve is close to the relay link, more power is allocated to jamming than to the desired signal, while the situation is reversed when Eve is far away. In particular, when Eve is $800 \mathrm{~m}$ away from the relay link, the power allocated to signal and jamming are the same, in which case the global power constraint would yield the same performance as with individual power constraints.

\section{CONCLUSIONS}

In this paper, we have proposed a cooperative jamming strategy for a two-hop relay system in the presence of an eavesdropper that can wiretap on both hops. In particular,

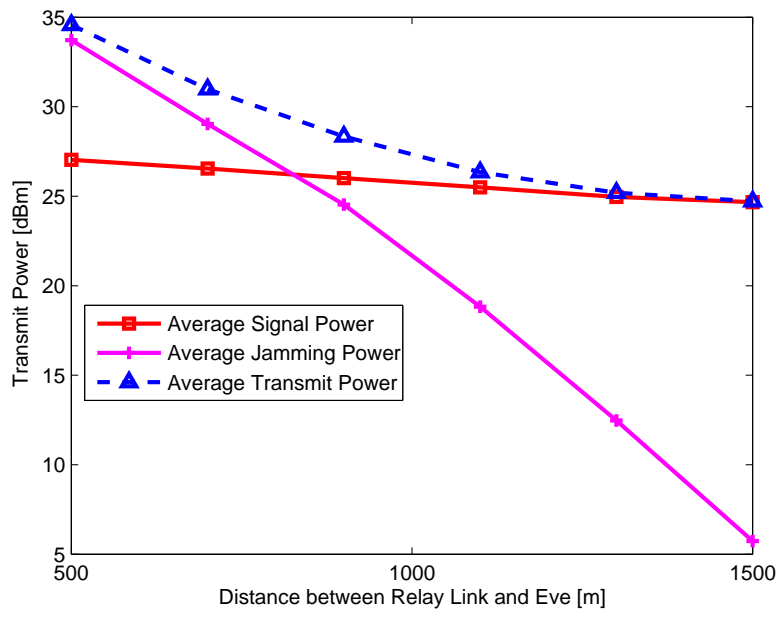

Fig. 4. Minimum transmit power vs. distance between Relay Link and Eve, $M=N=4, N_{e}=4, d_{a r}=d_{r b}=500 \mathrm{~m}, C_{0}=0.5 \mathrm{bps} / \mathrm{Hz}$.

we studied the joint design of the jamming beamformers and power allocation between jamming and the desired signal for the source, relay and destination. We showed that closed-form solutions for the beamformers are possible when using the suboptimal zero-forcing constraint that forces the jamming signals to be completely nulled at intended receivers. Based on this zero-forcing approach, we presented an optimal solution for power allocation using geometric programming. The cooperative jamming strategy is shown to provide a significant performance improvement compared with traditional relaying approaches in terms of secrecy capacity, especially when the eavesdropper is close to the relay link.

\section{REFERENCES}

[1] A. D. Wyner, "The wire-tap channel," Bell Systems Technical Journal, vol. 54, no. 8, pp. 1355-1387, Jan. 1975.

[2] F. Oggier and B. Hassibi, "The secrecy capacity of the MIMO wiretap channel," in Proc. IEEE International Symposium on Information Theory (ISIT 2008), Jul. 2008, pp. 524-528.

[3] A. Hero III, "Secure space-time communication," IEEE Trans. Inf. Theory, vol. 49, no. 12, pp. 3235-3249, Dec. 2003.

[4] S. Goel and R. Negi, "Guaranteeing secrecy using artificial noise," IEEE Trans. Wireless Commun., vol. 7, no. 6, pp. 2180-2189, Jun. 2008.

[5] A. L. Swindlehurst, "Fixed sinr solutions for the MIMO wiretap channel," in Proc. IEEE ICASSP 2009, Apr. 2009, pp. 2437-2440.

[6] A. Khisti, G. Wornell, A. Wiesel, and Y. Eldar, "On the gaussian MIMO wiretap channel," in Proc. IEEE ISIT 2007, Jun. 2007, pp. 2471-2475.

[7] Y. Oohama, "Capacity theorems for relay channels with confidential messages," in Proc. IEEE International Symposium on Information Theory (ISIT 2007), Jun. 2007, pp. 926-930.

[8] E. Tekin and A. Yener, "The general gaussian multiple-access and twoway wiretap channels: Achievable rates and cooperative jamming," IEEE Trans. Inf. Theory, vol. 54, no. 6, pp. 2735-2751, Jun. 2008.

[9] L. Lai and H. E. Gamal, "The relay-eavesdropper channel: Cooperation for secrecy," IEEE Trans. Inf. Theory, vol. 54, no. 9, pp. 4005-4019, Sep. 2008.

[10] L. Dong, Z. Han, A. P. Petropulu, and H. V. Poor, "Secure wireless communications via cooperation," in Proc. 46th Annual Allerton Conference on Communication, Control, and Computing, Sep. 2008, pp. 1132-1138.

[11] J. N. Laneman, D. Tse, and G. W. Wornell, "Cooperative diversity in wireless networks: Efficient protocols and outage behavior," IEEE Trans. Inf. Theory, vol. 50, no. 12, pp. 3062-3080, Dec. 2004.

[12] S. Boyd and L. Vandenberghe, Convex Optimization. Cambridge University Press, 2004. 